\title{
Contribución de la Terapia Ocupacional en la rehabilitación cardíaca: intervención, desafíos y reflexiones
}

\author{
Alba Vila Paz, Iván de Rosende Celeiro \\ Departamento de Ciencias de la Salud, Universidade da Coruña, A Coruña, España.
}

\begin{abstract}
Resumen: Introducción: La enfermedad cardiovascular aumenta la prevalencia de la discapacidad e incrementa la mortalidad en los países occidentales. En España, es el principal problema de salud de la población adulta. Objetivo: Conocer el rol profesional de la Terapia Ocupacional (TO) dentro de las actuaciones de rehabilitación cardíaca, en las diferentes fases del proceso de intervención, así como los instrumentos estandarizados disponibles actualmente para la evaluación. Método: Revisión y análisis narrativo de la literatura sobre TO y rehabilitación cardíaca. La base de datos de búsqueda en Medline no encontró ningún artículo; la base de datos IME, un estudio ha sido identificado. Además, se realizó una búsqueda manual de artículos, libros y otros trabajos académicos de relevancia en esta área de intervención. Resultados: Recuperar la independencia y autonomía en las ocupaciones y roles significativos es el objetivo principal de la TO. La intervención sigue un paradigma holístico, centrado en el logro de una vida activa y saludable, social y productiva, según las necesidades de cada persona. Se incluyen acciones individualizadas de asesoramiento y entrenamiento, incorporando los principios de conservación de energía, análisis ergonómico, simplificación de tareas y el uso de tecnología y adaptaciones del entorno. En la valoración del desempeño ocupacional, el terapeuta puede utilizar las escalas genéricas FIM, Barthel y COPM, al no existir herramientas específicas para esta población; el SF-36 es un instrumento destacado para analizar la calidad de vida. Conclusion: La relevancia de la TO en la promoción de la autonomía argumenta la necesidad de incorporar a este profesional en las estrategias de rehabilitación cardíaca, para favorecer enfoques educativos y prácticos centrados en la ocupación humana.
\end{abstract}

Palabras clave: Terapia Ocupacional, Cardiología, Calidad de Vida.

\section{Contribuiçáo da terapia ocupacional na reabilitaçáo cardíaca: intervençáo, desafios e reflexóes}

Resumo: Introdução: A doença cardiovascular aumenta a prevalência da deficiência e ocorre aumento da mortalidade nos países ocidentais. Na Espanha, é o principal problema de saúde da população adulta. Objetivo: Conhecer o papel do profissional da terapia ocupacional em ações de reabilitação cardíaca nas diferentes fases de intervenção e instrumentos padronizados atualmente disponíveis para avaliação. Método: Revisão e análise narrativa da literatura sobre terapia ocupacional e reabilitação cardíaca. A busca no banco de dados Medline não localizou nenhum artigo; na base de dados IME, um estudo foi identificado. Além disso, foi realizada uma busca manual de artigos, livros e outros trabalhos acadêmicos de relevância nesta área de intervenção. Resultados: Recuperar a independência e a autonomia em ocupações e papéis significativos constitui os principais objetivos da terapia ocupacional. A intervenção segue um paradigma holístico, centrado na obtenção de uma vida ativa e saudável, social e produtiva, de acordo com as necessidades de cada pessoa. Ações de aconselhamento e treinamento individualizado incluem a incorporação dos princípios de conservação de energia, a análise ergonômica, a simplificação de tarefas e o uso de tecnologia, e as adaptações ambientais. Na avaliação do desempenho ocupacional, o terapeuta ocupacional pode 
usar as escalas genéricas FIM, Barthel e COPM, na ausência de ferramentas específicas para essa população; SF-36 é um instrumento importante para se analisar a qualidade de vida. Conclusão: A relevância da terapia ocupacional na promoção da autonomia reforça a necessidade de envolver esse profissional nas estratégias de reabilitação cardíaca, para promover abordagens educacionais e práticas que incidem sobre a ocupação humana.

Palavras-chave: Terapia Ocupacional, Cardiologia, Qualidade de Vida.

\title{
Contribution of Occupational Therapy in cardiac rehabilitation: intervention, challenges and reflections
}

\begin{abstract}
Introduction: Cardiovascular disease increases the prevalence of disability and mortality in Western countries. In Spain, it is the main health problem of the adult population. Objective: To understand the professional role of Occupational Therapy in cardiac rehabilitation, in the different stages of intervention, as well as the standardized instruments currently available for evaluation. Method: Review and narrative analysis of the literature on Occupational Therapy and cardiac rehabilitation. The search in the Medline database did not locate any article; in the IME database, a study was identified. In addition, a manual search of articles, books and other academic works of relevance in this intervention area was conducted. Results: Regaining independence and autonomy in occupations and significant roles is the main goal of Occupational Therapy. The intervention follows a holistic paradigm, focused on achieving an active, healthy, social and productive life, according to the needs of each person. Individualized counselling and training actions include incorporating the principles of energy conservation, ergonomic analysis, simplification of tasks and the use of technology and environmental adaptations. In the evaluation of occupational performance, the therapist can use the generic scales FIM, Barthel and COPM in the absence of specific tools for this population; SF-36 is an important instrument to analyze quality of life. Conclusion: The relevance of OT in the promotion of autonomy argues the need to involve this professional in cardiac rehabilitation strategies, in order to promote educational and practical approaches focused on human occupation.
\end{abstract}

Keywords: Occupational Therapy, Cardiology, Quality of Life.

\section{Introdução}

\subsection{Enfermedad cardiovascular y rehabilitación cardíaca: un desafío para los servicios sanitarios y sociales occidentales}

Promover la autonomía, el bienestar y la calidad de vida en las personas con una enfermedad cardiovascular (ECV) es uno de los principales retos de las políticas sociosanitarias del presente siglo, en los países occidentales. El término ECV es ampliamente utilizado y permite definir el funcionamiento anormal y los trastornos del corazón y del sistema circulatorio; produce una situación de discapacidad, al presentar limitaciones de la actividad y restricciones en la participación, como consecuencia de la aparición de deficiencias en las funciones cardíacas, especialmente en relación a la capacidad de resistencia física. La prevención, el abordaje educativo y la rehabilitación cardíaca (RC) son estrategias destacadas para evitar la evolución de la ECV hacia la discapacidad, así como para reducir la morbimortalidad y la pérdida de calidad de vida en las personas con esta condición de salud, de especial prevalencia en los países desarrollados, en el contexto internacional actual.

Según la Organización Mundial de la Salud, las ECV, cerebrovasculares y otras dolencias cardíacas constituyen la primera causa de muerte en las sociedades industrializadas (WORLD..., 2014). La ECV es el principal problema de salud de la población adulta en España y ocupa el primer lugar como causa de muerte en las personas mayores de 45 años; en concreto, las ECV son responsables de más del 30\% de las defunciones (KOTSEVA et al., 2015). El resultado no sólo se cuantifica en datos de morbimortalidad; el impacto económico sobre los servicios sanitarios y sociales es creciente y, en consecuencia, las repercusiones socioeconómicas que generan se sitúan dentro de las principales preocupaciones y prioridades en la planificación de los servicios públicos (KOTSEVA et al., 2015). Según el European Cardiovascular Disease Statics 2012 (NICHOLS et al., 2012), el coste global derivado de la atención de esta condición de salud supone para los países de la Unión Europea un gasto superior a los 190 billones de euros anuales. 
La mortalidad, la morbilidad, así como las limitaciones en la capacidad funcional y en la participación social, en las personas con una ECV, se reducen con acciones de modificación del riesgo cardiovascular. Este abordaje incluye dos áreas de actuación principales: la prevención primaria, dirigida a la población sana, especialmente a las personas con alto riesgo coronario; y la prevención secundaria, cuyos objetivos son limitar el avance de la ECV y disminuir o evitar la discapacidad y la morbimortalidad (DE PABLO et al., 2008).

Existen una serie de factores de riesgo cardiovascular que explican la incidencia, la prevalencia y la severidad de la ECV. Los hábitos de conducta y estilos de vida insanos son factores que actúan como predictores de la enfermedad, antes de su debut clínico. El sexo, la edad o el factor hereditario, son elementos no modificables. También se ha demostrado que la personalidad está relacionada con el riesgo de cardiopatías (FERNÁNDEZ; MARTÍN; DOMÍNGUEZ, 2003). Sin embargo, existen otros factores de riesgo que sí son susceptibles de control, modificación y prevención, a través de acciones de educación sanitaria, RC y con la actuación interdisciplinar de profesionales como el terapeuta ocupacional (GRIMA et al., 2011; KOTSEVA et al., 2015; PACCI et al., 2011).

El objetivo principal de la RC es lograr el mayor grado posible de capacidad funcional cardíaca, con la finalidad de recuperar las actividades diarias, fomentando el retorno a una vida productiva, saludable y significativa, desde la perspectiva subjetiva de cada persona. Se incluyen otros objetivos secundarios, adaptados durante todo el proceso de intervención. A corto plazo, se intenta reanudar las actividades cotidianas de manera inmediata, facilitar apoyo psicológico y proporcionar instrucciones para favorecer el proceso de recuperación tras el accidente cardíaco. Los objetivos a largo plazo abarcan la modificación de los factores de riesgo cardiovascular o de hábitos que puedan favorecer la progresión de la enfermedad e influir en el pronóstico de la misma, especialmente a través de estrategias de carácter educativo y estimulando su incorporación en la vida diaria (SANTOS; DE JUAN, 2006; MIRANDA, 2010; PASHKOW, 1991).

Los programas de RC están integrados en un proceso de atención global y se encuentran influidos de una manera relevante por las características de la valoración clínica multidisciplinar, el tratamiento médico, la evaluación de los factores de riesgo modificables, así como, dentro del ámbito propio de la Terapia Ocupacional, el análisis de las barreras y facilitadores que encuentra el usuario en el desempeño ocupacional. La American Association of Cardiovascular and Pulmonary Rehabilitation (BALADY et al., 2000) establece que los principales componentes de las actuaciones de RC son reducir el riesgo cardiovascular, adquirir comportamientos saludables, disminuir la discapacidad y promover un estilo de vida activo.

En la actualidad, los programas de RC son multifactoriales, es decir, se combina el entrenamiento físico y estrategias de áreas específicas como la intervención psicológica, la educación para la salud y los programas de Terapia Ocupacional. Los principios clave de este tipo de intervención son el tratamiento individualizado, el trabajo en equipo de los profesionales, la consideración conjunta de las alteraciones físicas, emocionales y sociales, así como la intervención a nivel individual, grupal y comunitario (SANTOS; DE JUAN, 2006).

Según la Sociedad de Rehabilitación CardioRespiratoria (GÓMEZ et al., 2015), entidad española de relevancia en el ámbito de estudio, un programa de $\mathrm{RC}$ eficaz debe ser un proceso integral que facilite el retorno a la vida comunitaria y promueva el conocimiento, la aceptación y la integración, en la vida diaria, de estrategias dirigidas a minimizar el riesgo de eventos cardíacos futuros, a través de las pautas y hábitos propios del estilo de vida cardiosaludable. Conocer e implementar estas estrategias en las ocupaciones cotidianas conlleva un proceso estructurado de manera interdisciplinar y el terapeuta ocupacional es un profesional con un rol destacado en el área educativa, focalizada en la formación y el asesoramiento, así como en las actuaciones de entrenamiento práctico para recuperar los roles significativos en la vida diaria de la persona.

El objetivo de la presente revisión consiste en describir la producción empírica y conceptual sobre la intervención de Terapia Ocupacional en los programas de $\mathrm{RC}$, a partir de estudios científicos y documentos académicos, para proporcionar una visión actualizada sobre los siguientes aspectos de la práctica clínica y comunitaria de este profesional: objetivos de la intervención, proceso y etapas, actuaciones y herramientas que utiliza en cada una de las etapas de la RC, así como instrumentos de valoración para la práctica profesional del terapeuta ocupacional. Los resultados aportarán información científica de interés como herramienta para la planificación de intervenciones de Terapia Ocupacional en personas con enfermedad con una ECV y para futuras investigaciones en este ámbito de actuación. 


\section{Metodo}

El tipo de investigación es la revisión narrativa. Los criterios de inclusión utilizados para la selección de artículos y documentos académicos son: a) Artículos que describen y/o analizan las siguientes áreas temáticas en relación a la intervención de Terapia Ocupacional en la RC: objetivos de la práctica profesional, etapas del proceso, actuaciones o herramientas que utiliza este profesional para el trabajo en el ámbito estudiado, así como instrumentos de evaluación de interés para su uso en la práctica diaria; b) estudios originales (cualitativos, cuantitativos, combinados y/o revisiones bibliográficas), literatura gris y editoriales; c) publicaciones escritas en inglés o en español; d) publicaciones que analizan o describen una intervención de Terapia Ocupacional en el colectivo de personas adultas (mayores de 18 años de edad). Los límites en relación al ámbito temporal de publicación de los documentos se describen a continuación, al recoger los detalles de cada búsqueda bibliográfica realizada. Para la localización de estudios científicos en la base de datos Medline, se utilizaron las siguientes palabras clave: "Occupational Therapy", "Cardiac rehabilitation" y "Heart diseases". La estrategia de búsqueda fue: MH "Occupational Therapy" AND TX "Cardiac rehabilitation" OR MH "Heart diseases". El período incluido engloba todos los documentos publicados entre los años 2000 y 2015. Como resultados de la búsqueda, se localizó un total de 6 artículos: uno de ellos fue excluido por criterio de edad (estudio en población menor de 18 años); los restantes se excluyeron por no cumplir con los objetivos de la presente revisión. Se realizó otra búsqueda de estudios científicos en la base de datos Índice Médico Español (IME), dependiente del Consejo Superior de Investigaciones Científicas (CSIC) español. Esta base de datos recopila información científica del ámbito de las Ciencias de la Salud, a partir de estudios publicados en España. Las palabras clave empleadas fueron: "Terapia Ocupacional" y "Rehabilitación Cardíaca", combinadas con el operador AND. El período analizado incluye todos los documentos publicados entre los años 2000 y 2015. Se localizó un estudio (MARTÍNEZ; PÉREZ DE HEREDIA; MIANGOLARRA, 2002), incluido al cumplir con los criterios establecidos en la presente revisión.

Una vez consultadas estas dos bases de datos bibliográficas, y como consecuencia del limitado número de estudios localizado, se realizaron búsquedas manuales en monografías, libros, documentos técnicos y otros trabajos académicos considerados de relevancia para el tema de estudio, con un período temporal mayor: publicación entre los años 1995 y
2015. También se llevaron a cabo búsquedas con la técnica bola de nieve (snowballing), revisando las listas de referencias de los artículos ya incluidos para revisión en este trabajo, con el objetivo de verificar la existencia de artículos adicionales no emergentes en las bases de datos. El análisis de los resultados consistió en la implementación de una estrategia de análisis temático, de acuerdo a los objetivos planteados en la presente investigación. Se agruparon los documentos analizados por temas, para aportar una visión clara y global de los resultados obtenidos, incluyendo 3 categorías temáticas generales: aspectos generales de la intervención de Terapia Ocupacional en la RC, haciendo mayor hincapié en los objetivos generales de la actuación profesional (primer apartado de los Resultados); etapas del proceso de intervención de Terapia Ocupacional en el ámbito de la RC (segundo apartado); y, por último, herramientas de valoración de interés para la práctica clínica y comunitaria de la Terapia Ocupacional en RC.

\section{Resultados e Discusión}

\subsection{Terapia Ocupacional y rehabilitación cardíaca: una profesión clave en el logro de la autonomía, la participación social y la calidad de vida}

Alcanzar la máxima independencia funcional y, en consecuencia, la autonomía en las ocupaciones significativas, la participación comunitaria y la calidad de vida, son los principales objetivos de la Terapia Ocupacional. La incorporación de este profesional en los equipos interdisciplinares de RC ha aumentado de forma progresiva en los últimos años en diversos países occidentales, aunque continúa siendo un reto y un desafío en Espańa, en los servicios dirigidos a abordar las necesidades de salud y bienestar de este grupo de población creciente.

Durante el siglo XX, una proporción amplia de los terapeutas ocupacionales que desempeńaban su rol profesional en RC planificaban su intervención desde un modelo biomecánico, empleando actividades graduadas y pesos estructurados para simular situaciones reales de la vida diaria. Este tipo de tratamiento ha ido evolucionando y, actualmente, la Terapia Ocupacional sigue un paradigma holístico, centrado en el logro de una vida activa, social y productiva, acorde a las necesidades y prioridades de cada persona. El objetivo principal es lograr la máxima autonomía e independencia en el desempeño de las actividades y roles cotidianos, así como recuperar las ocupaciones significativas para el usuario. 
La valoración del desempeño ocupacional, el asesoramiento sobre las actividades de la vida diaria (AVD), así como la adaptación funcional de la actividad, son las principales herramientas del terapeuta ocupacional. La intervención analiza e incorpora, mediante actuaciones individualizadas, el potencial terapéutico de los principios de conservación de energía, el análisis ergonómico de la actividad, la simplificación de tareas y el uso de los productos de apoyo y de las adaptaciones del entorno (GÓMEZ et al., 2010; CASTRO et al., 2003; OLIVER; SEWELL, 2003; SOSA, 1998).

Las técnicas de conservación de energía se dirigen a disminuir la aparición de fatiga, disnea y la percepción de esfuerzo, en personas con una limitación de la capacidad de resistencia física, mediante una organización y planificación de las actividades a realizar durante un periodo de tiempo o en la vida diaria. El terapeuta ocupacional promoverá el empleo de la tecnología de apoyo (ej. asiento o banco de transferencia en ducha/bañera, alza y barra de apoyo en inodoro, tecnología motorizada para facilitar actividades productivas, etc.), la eliminación de pasos innecesarios en las tareas y fomentará la inclusión de periodos de descanso y la alternancia entre los tiempos de trabajo/actividad y reposo. Para evitar el sobreesfuerzo y el desarrollo de fatiga, es importante limitar la realización de movimientos corporales repetitivos, la manipulación de cargas pesadas o el desarrollo de actividades contra la acción de la gravedad.

Este profesional evalúa las demandas de la actividad, el contexto y las características, deseos y preferencias de la persona, en relación a las ocupaciones y la vida comunitaria. Cuando la actividad requiere un nivel de esfuerzo al que la persona no logra dar respuesta, y para evitar que una situación de dependencia se instaure o agrave, la actuación de Terapia Ocupacional adapta las ocupaciones significativas e incluye un entrenamiento de estas actividades en el entorno social de convivencia y desarrollo de la persona (DUNLAY et al., 2015), progresivo y con la participación activa del usuario y de las personas relevantes de su contexto.

El asesoramiento y las actuaciones de entrenamiento práctico favorecen el ahorro energético y la ergonomía en el desempeño diario. La intervención se dirige a identificar y adaptar ocupaciones que puedan conferir un riesgo evitable (OLIVER; SEWELL, 2003); por ello, el rol del terapeuta ocupacional es imprescindible, al considerar aspectos de relevancia para la satisfacción y la calidad de vida y complementarios a la intervención médica y clínica convencional. La Terapia Ocupacional evalúa la necesidad de modificar el estilo de vida y se focaliza en la adaptación de las tareas diarias, para maximizar la independencia funcional; en concreto, esta profesión dirige su actuación prioritariamente hacia la autonomía en el desempeño de las actividades de autocuidado, la reincorporación laboral y las ocupaciones de ocio, mediante el desarrollo de un plan de tratamiento basado en el estilo de vida, rutinas, profesión y roles de cada persona (ÁVILA et al., 2010).

\subsection{Proceso de intervención de Terapia Ocupacional en los programas de rehabilitación cardíaca}

Tradicionalmente, los programas de RC se han estructurado en tres fases, de duración variable y con una implementación a lo largo de un proceso continuo y dinámico. A continuación, se describen las características y actuaciones principales de cada etapa, así como el rol del terapeuta ocupacional a lo largo de este proceso de intervención. La Tabla 1 resume las características de las tres fases de intervención.

Tabla 1. Breve resumen de las fases de RC.

\begin{tabular}{|c|c|c|}
\hline & Duración estimada & Referencia bibliográfica \\
\hline Fase aguda (I) & $\begin{array}{l}\text { 8-12 días. } \\
\text { Movilización precoz. } \\
\text { Ejercicios graduados a baja resistencia. } \\
\text { Inicio de programa de actividades de la vida diaria: } \\
\text { autocuidado, movilidad en entornos contralados, trabajo de } \\
\text { hábitos nocivos. }\end{array}$ & $\begin{array}{l}\text { Martínez, Pérez de Heredia y } \\
\text { Miangolarra (2002), Santos y } \\
\text { De Juan (2006) }\end{array}$ \\
\hline $\begin{array}{c}\text { Fase de } \\
\text { convalecencia o } \\
\text { etapa subaguda } \\
\text { (II) }\end{array}$ & $\begin{array}{l}4-5 \text { días o } 3 \text { a } 6 \text { meses. } \\
\text { Educación del paciente y su familia con sesiones individuales } \\
\text { o grupales. } \\
\text { Fomento de conductas saludables. } \\
\text { Disfunción sexual. } \\
\text { Reincorporación laboral. }\end{array}$ & $\begin{array}{l}\text { Martínez, Pérez de Heredia y } \\
\text { Miangolarra (2002), Santos } \\
\text { y De Juan (2006), Miranda } \\
\text { (2010), Wilke y Wilke (2002) }\end{array}$ \\
\hline $\begin{array}{c}\text { Fase de } \\
\text { mantenimiento } \\
\text { (III) }\end{array}$ & $\begin{array}{l}\text { Mantenimiento del estilo de vida adquirido en los estadios } \\
\text { I y II. }\end{array}$ & $\begin{array}{l}\text { Wilke y Wilke (2002), } \\
\text { Miranda (2010) }\end{array}$ \\
\hline
\end{tabular}


La fase I se desarrolla durante la estancia hospitalaria, con una duración aproximada de 8 a 12 días. En la actualidad, se registra una tendencia creciente en los servicios sanitarios a reducir en lo posible la duración del ingreso hospitalario por una ECV. El objetivo prioritario del terapeuta ocupacional en esta etapa consiste en reeducar el desempeño de las actividades de autocuidado, con la finalidad de recuperar la autonomía en las ocupaciones básicas; favorecer una movilización precoz y progresiva, para contrarrestar los efectos nocivos de la inactividad y evitar la pérdida de masa muscular durante el período de reposo; así como iniciar las acciones de sensibilización y concienciación sobre la importancia de disminuir los factores de riesgo cardiovascular modificables.

El terapeuta ocupacional inicia el programa de AVD tras la estabilización del proceso agudo y la situación clínica. La intervención es progresiva y engloba acciones de educación para el conocimiento de la ECV, entrenamiento de la independencia en las ocupaciones básicas, adaptado a partir de los principios de conservación de energía, ergonomía y simplificación de tareas, así como actividades de movilidad en un entorno controlado, formado principalmente por la habitación, el cuarto de baño y, posteriormente, el pasillo y otras áreas del contexto hospitalario. La deambulación se inicia tan pronto como la persona se encuentre estable. Las tareas de autocuidado a evaluar y entrenar serán las relacionadas con la alimentación, el vestido (especialmente la preparación de la ropa y vestido de la parte inferior), el aseo personal básico (lavar las manos y la cara, peinarse, cortar uńas, afeitado, etc.), las transferencias corporales (movilidad en decúbito, levantarse de la cama, paso de sedestación a bipedestación, etc.), la regulación de la micción/defecación (desplazamiento y transferencia al inodoro, vestido/desvestido, higiene personal y manejo de dispositivos relacionados con la incontinencia, cuando sea necesario) y las actividades de lavar la parte superior e inferior del cuerpo. Un indicador de evolución favorable en el ámbito de la autonomía personal, durante la fase de estancia hospitalaria, puede ser el desempeño autónomo y seguro de la actividad de ducha, al requerir las mayores demandas de consumo energético, así como la independencia en el desplazamiento por el entorno hospitalario. Durante el proceso de intervención, el terapeuta ocupacional promueve el empleo de productos de apoyo y asesora sobre las necesidades de adaptación funcional del hogar, con la finalidad de favorecer el alta hospitalaria a un contexto seguro y facilitador de la independencia.
Es fundamental evaluar las demandas de cada actividad, ordenarlas en una secuencia y estimular su desempeño de una forma progresiva, en función de la evolución de cada persona a nivel físico y emocional. Cualquier tarea con propósito exige una demanda de energía sobre el sistema cardiopulmonar. El gasto de energía en las personas con una ECV se mide mediante el equivalente metabólico, conocido con el término de MET. El MET es la energía que se gasta en un estado de reposo (AINSWORTH et al., 2000); 1 MET equivale a un consumo de $3,5 \mathrm{ml}$ de oxígeno por kilogramo de peso corporal y por minuto. Las AVD se evalúan sobre la base del MET en reposo. Una actividad cardíaca mínima o muy leve requiere aproximadamente 1-2 METs; una actividad leve, entre 2 y 3 METs; moderada, cuando supone un gasto energético equivalente a 3-4 METs; se considera actividad intensa cuando el consumo de energía supera los 4-5 METs. Si la capacidad física del usuario es superior a los 7 METs de trabajo, el riesgo de la persona se evalúa como "bajo" (MAROTO; PRADOS, 2010). Dada su relevancia dentro de los objetivos de la RC, es importante tener en cuenta que el aumento de un MET en la capacidad funcional se relaciona con una reducción del 15\% en las tasas de mortalidad cardiovascular (GÓMEZ et al., 2015). En la Tabla 2 se detallan ejemplos de los equivalentes metabólicos de las actividades diarias, con respecto a la intensidad de las AVD y el gasto energético.

Durante esta etapa, el terapeuta ocupacional realiza un asesoramiento sobre las ocupaciones de ocio, para promover el uso significativo del tiempo libre y, de esta forma, contribuir en la disminución del estrés y de la ansiedad. Se analiza qué actividades lúdicas puede comenzar a realizar, la secuencia, los tiempos y cómo se puede adaptar cada actividad a la capacidad funcional de la persona. La intervención durante la fase hospitalaria se complementa con el

Tabla 2. Equivalentes metabólicos de las actividades de la vida diaria.

\begin{tabular}{lcc}
\hline \multicolumn{1}{c}{ Ocupaciones } & Intensidad & MET \\
\hline Comer solo en la cama & Muy leve & 1 \\
Andar o pasear a 3-4 km/h & Leve & 2,5 \\
Higiene personal en & Leve & 2,5 \\
bipedestación & & \\
Vestido independiente & Moderada & 3 \\
Ducha en sedestación & Intensa & 4 \\
Subir escaleras & Intensa & 4,3 \\
Desempeño del autocuidado & Intensa & $>5$ \\
con independencia & & \\
Actividad sexual & & \\
\hline
\end{tabular}

Fuente: Ainsworth et al. (2000). 
inicio de las actuaciones educativas de asesoramiento sobre los factores de riesgo cardiovascular, los beneficios de la actividad física/lúdica y las relaciones sociales, así como la importancia de modificar el estilo de vida, con el objetivo de promover hábitos cardiosaludables (WILKE; WILKE, 2002). Se trata de acciones formativas con un carácter de iniciación e introductorio, dado que este tipo de estrategias serán las predominantes en las fases II y III de la RC.

La fase II incluye el desarrollo de un programa multidisciplinar, a implementar en unidades específicas de RC, con un ingreso medio de 4 a 5 semanas, y/o de una forma ambulatoria durante un tiempo aproximado de 3 a 6 meses. El abordaje rehabilitador tiene como finalidad, en esta etapa, desarrollar y mantener el máximo nivel de capacidad funcional posible. El terapeuta ocupacional valora, asesora y entrena a la persona en el desempeño de las ocupaciones instrumentales, laborales y de ocio significativas, considerando en cada momento la capacidad cardíaca (en METs), así como los intereses, roles deseados y prioridades de cada usuario. Con la participación activa de la persona, se diseña un programa individualizado, orientado a la recuperación, mantenimiento y adquisición de actividades, incorporándolas en la rutina diaria, semanal y mensual de la persona, de una forma progresiva. El asesoramiento sobre las necesidades ocupacionales incluye acciones de formación y entrenamiento en el uso de productos de apoyo, adaptaciones del entorno y el conocimiento y aplicación de los principios de ergonomía y conservación de la energía, en el desempeño diario (MAROTO; PRADOS, 2010).

En relación a la adaptación de las actividades laborales, será necesario evaluar las demandas físicas de la actividad productiva desarrollada previamente, así como las preferencias del usuario en este ámbito. Cuando las tareas del trabajo superan la capacidad funcional de la persona, se planteará la adaptación del puesto de trabajo o el asesoramiento y exploración de nuevas actividades laborales significativas. En el proceso de ajuste entre las capacidades de la persona y las demandas de las tareas laborales, el terapeuta ocupacional analizará los espacios de trabajo, para promover la accesibilidad y el uso de tecnología como grúas, cintas transportadoras, carros, apoyos isquiáticos para el descanso en tareas de bipedestación prolongada, etc. Se estudiará la disposición de los diferentes materiales a emplear, para situarlos dentro del plano funcional de alcance de la persona, desde la posición de trabajo necesaria (sedestación, bipedestación o, preferentemente, la alternancia entre diversas posturas corporales). Las herramientas de la actividad laboral serán ligeras y se limitará la manipulación o el traslado de pesos.

La intervención ocupacional incluye acciones educativas dirigidas hacia la persona y a su entorno cercano (familia y red social de apoyo informal), realizadas mediante entrevistas personales y sesiones individuales o grupales. No sólo se ejecutan estrategias educativas, sino que será fundamental planificar y practicar el desempeńo de las ocupaciones significativas, en el contexto domiciliario, laboral y comunitario de la persona. El entrenamiento incluye materiales informativos, registros de actividades y pautas para el desempeño de actividades, así como acciones de feedback sobre los resultados obtenidos. La retroalimentación se dirige a mejorar la autoestima y a evitar la aparición de trastornos emocionales, mejorando la confianza del usuario en sus propias capacidades y potencial. En resumen, durante la intervención de Terapia Ocupacional, se aporta al usuario información y experiencia amplia sobre las ocupaciones que puede desempeñar de una forma autónoma, segura y satisfactoria, atendiendo a la capacidad de esfuerzo adquirida; se analizan los contextos y adaptaciones y, por último, se planifica el desarrollo de un estilo de vida activo, caracterizado por el equilibrio de diversas ocupaciones con significado y relevancia para la persona (básicas, instrumentales, productivas, de ocio y participación comunitaria).

La fase III se focaliza en la implementación de acciones de prevención, para disminuir los factores de riesgo cardiovascular, de una forma permanente en la vida diaria, así como en el mantenimiento, a largo plazo, del estilo de vida saludable desarrollado en los estadios I y II de RC. Las estrategias contemporáneas de $\mathrm{RC}$ recomiendan el desarrollo de las actuaciones de esta etapa mediante una estrecha colaboración con los recursos comunitarios, sanitarios o sociales (centros de salud, centros sociales y entidades del ámbito asociativo). Las actividades físicas y ocupaciones deportivas se incorporan en el entrenamiento cardiovascular, como tareas de mantenimiento, pero sin sobrepasar la frecuencia cardíaca de seguridad permitida, en función de la capacidad funcional de cada persona. Las actividades se recomiendan a partir de la entrevista con la persona e indicando tipologías, pautas, intensidad y frecuencia de cada tarea. El terapeuta ocupacional realizará un seguimiento individualizado de las ocupaciones de ocio, laborales y de participación social del usuario, para evaluar la posibilidad de incorporar nuevos intereses o planificar adaptaciones y ajustes dirigidos a mantener la máxima funcionalidad y satisfacción en el desempeño diario. La American Heart Association recomienda que la fase III de la RC 
Tabla 3. Herramientas para la valoración en personas con una enfermedad cardiovascular.

\begin{tabular}{|c|c|c|}
\hline $\begin{array}{c}\text { Desempeño de las actividades de } \\
\text { la vida diaria }\end{array}$ & \multicolumn{2}{|c|}{ Calidad de vida } \\
\hline Genéricos & Genéricos & Específicos \\
\hline $\begin{array}{l}\text { Functional Independence Measure } \\
\text { (FIM) }\end{array}$ & $\begin{array}{l}\text { Cuestionario de Salud Short Form } \\
\text { (SF-36) }\end{array}$ & $\begin{array}{l}\text { Quality of Life Index-Cardiac } \\
\text { Version }\end{array}$ \\
\hline Índice de Barthel & Nottingham Health Profile & $\begin{array}{l}\text { Cuestionario Español de Calidad } \\
\text { de Vida en pacientes post-infarto }\end{array}$ \\
\hline Escala de Lawton y Brody & Sickness Impact Profile (SIP) & $\begin{array}{l}\text { Angina Pectoris Quality of Life } \\
\text { Questionnaire }\end{array}$ \\
\hline $\begin{array}{l}\text { Canadian Occupational } \\
\text { Performance Measure (COPM) }\end{array}$ & & $\begin{array}{l}\text { Quality of Life after Myocardial } \\
\text { Infarction }\end{array}$ \\
\hline
\end{tabular}

Fuente: Law et al. (1998), Grima et al. (2011).

se prolongue durante toda la vida cuando existen factores de riesgo cardiovascular, comorbilidad y una elevada posibilidad de eventos cardíacos posteriores (BITTNER, 2012).

\subsection{Terapia Ocupacional y rehabilitación cardíaca: instrumentos para la valoración}

De una forma previa a la planificación del programa de intervención, el terapeuta ocupacional realiza una valoración detallada de las áreas de interés para la actuación profesional. A través de la revisión de los informes de salud y sociales, así como la entrevista con el usuario y personas significativas de su contexto, se recoge información sobre la historia de vida previa de la persona, otros antecedentes, la evolución del proceso cardíaco, la estratificación del riesgo, el desempeño ocupacional, así como las prioridades e intereses del usuario, en relación a la intervención de Terapia Ocupacional.

En los ámbitos clínico y comunitario, así como en un contexto investigador, el terapeuta ocupacional puede utilizar herramientas estandarizadas de evaluación, para estructurar y facilitar la recogida de información. Detallar los resultados de la aplicación de estos instrumentos, antes (línea base) y después de la intervención, permite evaluar y aportar evidencia científica sobre la eficacia de la actuación del terapeuta ocupacional. Las herramientas de evaluación de mayor interés para la Terapia Ocupacional son las dirigidas a evaluar el desempeño de las ocupaciones significativas y el nivel de calidad de vida adquirido a lo largo del proceso de intervención.

La Tabla 3 recoge los principales instrumentos estandarizados de evaluación de las AVD y de la calidad de vida en personas con una ECV. No se han localizado escalas de valoración del grado de independencia en las AVD específicas del ámbito de cardiología; por ello, el terapeuta ocupacional puede utilizar instrumentos genéricos, válidos para diversos tipos de poblaciones y diferentes condiciones de salud. La Functional Independence Measure (FIM) y el Índice de Barthel proporcionan información sobre el desempeño en las AVD básicas. La independencia en AVD instrumentales puede valorarse mediante la escala de Lawton y Brody. Con los objetivos de conocer las ocupaciones prioritarias para cada persona, así como para favorecer la autoevaluación en relación al desempeño de las actividades significativas para el usuario, se recomienda el instrumento Canadian Occupational Performance Measure (COPM), desarrollado por (LAW et al., 1998) dentro del Modelo de Práctica Canadiense y ampliamente utilizado en el contexto internacional, en la práctica profesional de la Terapia Ocupacional.

Con respecto a los instrumentos de la calidad de vida más empleados en RC, diferentes guías clínicas coinciden en que la escala SF-36 es el cuestionario más adecuado para evaluar la calidad de vida en la población de estudio. No existe consenso sobre las herramientas específicas, aunque estos cuestionarios son más sensibles para detectar los cambios e incluyen dimensiones concretas de la enfermedad cardíaca, de interés para la planificación de las intervenciones de RC.

\section{Conclusiones}

La principal aportación de la presente revisión consiste en el análisis de la información científica y actualizada disponible sobre la práctica de la Terapia Ocupacional en un ámbito de actuación novedoso, con una escasa implantación a nivel internacional. Sin embargo, en coherencia con la limitada presencia de este profesional en la RC, esta revisión ha identificado un nivel de información científica muy reducido, argumentando la necesidad de plantear investigaciones, documentos de consenso y guías de práctica clínica para guiar el desarrollo de actuaciones de Terapia Ocupacional en este campo. 
$\mathrm{La} \mathrm{RC}$ es una de las estrategias prioritarias en las políticas sanitarias y sociales para las personas con ECV, grupo de población con necesidades de intervención de especial prevalencia en los países occidentales. El terapeuta ocupacional desempeña un papel fundamental en el proceso de recuperación de las ocupaciones y roles significativos para la persona; este profesional favorece el desarrollo de un estilo de vida activo y saludable, y, por lo tanto, promueve el bienestar y la calidad de vida. El retorno a las actividades cotidianas debe ser un proceso individualizado, gradual y progresivo, dirigido a adquirir y mantener habilidades de independencia en el desempeño diario, disminuir el impacto de la ECV a nivel físico, mental y social, modificar el estilo de vida y reducir los factores de riesgo cardiovascular. A pesar de su rol destacado en la promoción de la autonomía, la implementación de estrategias de Terapia Ocupacional continúa siendo muy reducida en el ámbito de la cardiología; por ello, y con el objetivo de desarrollar enfoques educativos y prácticos centrados en la ocupación humana, es necesario incorporar a este profesional en los equipos interdisciplinares de intervención en personas con ECV.

\section{Referencias}

AINSWORTH, B. E. et al. Compendium of physical activities: an update of activity codes and MET intensities. Medicine and Science in Sports and Exercise, Hagerstown, v. 9, n. 32, p. 498-504, 2000.

ÁVILA, A. A. et al. Marco de trabajo para la práctica de la Terapia Ocupacional: dominio y proceso. Puerto Rico: Asociación Americana de Terapia Ocupacional, 2010.

BALADY, G. J. et al. Core components of cardiac rehabilitation/secondary prevention programs: a statement for healthcare professionals from the American Heart Association and the American Association of Cardiovascular and Pulmonary Rehabilitation Writing Group. Circulation, United States, v. 102, n. 9, p. 1069-1073, 2000.

BITTNER, V. Cardiac rehabilitation: call to action for healthcare providers. Circulation, Baltimore, v. 126, p. 671-673, 2012.

CASTRO, B. A. et al. Plan integral de Cardiopatía Isquémica 2004-2007. Madrid: Ministerio de Sanidad y Consumo, 2003.

DE PABLO, C. et al. Prevención cardiovascular y rehabilitación cardiaca. Revista Española de Cardiologia, Madrid, v. 61, n. 1, p. 97-108, 2008.

DUNLAY, S. M. et al. Activities of daily living and outcomes in heart failure. Circulation: Heart Failure, Greenville, v. 8, n. 2, p. 261-267, 2015.
FERNÁNDEZ, A. E.; MARTÍN, M. D.; DOMÍNGUEZ, F. J. Factores de riesgo e intervenciones psicológicas eficaces en los trastornos cardiovasculares. Psicothema, Oviedo, v. 15, n. 4, p. 615-630, 2003.

GÓMEZ, A. M. et al. Equipo multidisciplinario en las Unidades de Rehabilitación cardíaca. ¿Qué papel desempeñamos? In: PLEGUEZUELOS, E. et al. Principios de rehabilitación cardíaca. Madrid: Panamericana, 2010. p. 127-136.

GÓMEZ, A. et al. Recomendaciones sobre rehabilitación cardíaca en la cardiopatía isquémica de la Sociedad de Rehabilitación Cardio-Respiratoria. Rehabilitación, Madrid, v. 49, n. 2, p. 102-124, 2015.

GRIMA, A. et al. Cardiología preventiva y rehabilitación cardiaca. Revista Española de Cardiología, Madrid, v. 64, n. 1, p. 66-72, 2011.

KOTSEVA, K. et al. EUROASPIRE IV: A European Society of Cardiology survey on the lifestyle, risk factor and therapeutic management of coronary patients from 24 European countries. European Journal of Preventive Cardiology, London, v. 1, n. 1, p. 1-13, 2015.

LAW, M. et al. Canadian occupational performance measure. Otawa: CAOT, 1998.

MAROTO, J. M.; PRADOS, C. Rehabilitación cardíaca: historia, indicaciones, protocolos. In: MAROTO, J.; DE PABLO, C. Rehabilitación cardiovascular. Madrid: Panamericana, 2010. p. 3-16.

MARTÍNEZ, R. M.; PÉREZ DE HEREDIA, M.; MIANGOLARRA, J. C. Terapia ocupacional en los programas de rehabilitación cardíaca. Rehabilitación, Madrid, v. 36, n. 4, p. 227-234, 2002.

MIRANDA, G. Indicaciones de los programas de rehabilitación cardiovascular. In: PLEGUEZUELOS, E. et al. Principios de rehabilitación cardíaca. Madrid: Panamericana, 2010. p. 137-147.

NICHOLS, M. et al. European cardiovascular disease statistics. Brussels: European Society of Cardiology, 2012.

OLIVER, K.; SEWELL, L. Enfermedad cardíaca y respiratoria. In:TURNER, A.; FOSTER, M.; JOHNSON, S. Terapia ocupacional y disfunción fisica: principios, técnicas y práctica. Madrid: Elservier, 2003. p. 581-597.

PACCI, K. et al. Impacto de la rehabilitación cardiaca en la calidad de vida de los pacientes del Instituto Nacional Cardiovascular del Perú. Revista Peruana de Epidemiología, Lima, v. 15, n. 2, p. 126-129, 2011.

PASHKOW, F. J. Rehabilitation strategies for the complex cardiac patient. Cleveland Clinic Journal of Medicine, Cleveland, v. 58, n. 1, p. 70-75, 1991.

SANTOS, J. F.; DE JUAN, J. Rehabilitación cardíaca. In: SÁNCHEZ, I. et al. Manual SERMEF de rehabilitación y medicina fisica. Madrid: Panamericana, 2006. p. 679-740. 
SOSA, V. Estudio de la incapacidad laboral por enfermedades cardiocirculatorias. Madrid: Instituto Nacional de Medicina y Seguridad del Trabajo, 1998.

WILKE, M.; WILKE, A. Aspectos generales y resultados de la rehabilitación cardiaca. In: ESPINOSA, J.;
BRAVO, N. Rehabilitación cardíaca y atención primaria. Madrid: Panamericana, 2002. p. 25-60.

WORLD HEALTH ORGANIZATION - WHO. Needs and action priorities in cardiac rehabilitation and secondary prevention in patients with CHD. Ginebra, 2014.

\section{Contribución de los Autores}

Ambos autores han sido responsables de la elaboración, revisión y aprobó la versión final del texto. 